Original article

Agronomy

\title{
Estimation du stock de carbone dans les faciès arborés et arbustifs des savanes soudano-guinéennes de Ngaoundéré, Cameroun.
}

\author{
Adamou IBRAHIMA ${ }^{1^{*}}$ et Chimène ABIB FANTA2
}

1Département des Sciences Biologiques, Faculté des Sciences, Université de Ngaoundéré, B.P. 454

'Département des Sciences Alimentaires et de Nutrition, ENSAI, Université de Ngaoundéré, B.P. 455, Ngaoundéré, Cameroun.

* correspondance (aibrahima@hotmail.com)

\begin{abstract}
RESUME
Une des conséquences environnementales de la dégradation forestière est la perte de carbone, qui à son tour contribue indirectement au changement climatique. Dans la perspective d'évaluer la contribution des savanes de l'Adamaoua dans le changement climatique, une étude portant sur l'estimation de stock du carbone a été menée dans les faciès arbustifs et arborés. La quantité de carbone a été estimée dans des parcelles carrées de $100 \mathrm{~m}^{2}$ pour les arbres et les arbustes, de $1 \mathrm{~m}^{2}$ pour le sous-bois et de $0,0625 \mathrm{~m}^{2}$ pour les racines et le sol. Le dispositif expérimental est un bloc complètement randomisé à trois répétitions. Les types de savanes sont des traitements principaux et les parcelles les répétitions. Les résultats montrent que la quantité totale de carbone est de l'ordre de 81,48 et 118,36 tC/ha respectivement pour la savane arbustive et arborée. Dans la savane arborée, l'essentiel de carbone est stocké dans la phytomasse des arbres (65,30 tC/ha) et dans le sol (48,37 tC/ha); la contribution des arbustes (3,83t/ha) des herbacées $(0,30 \mathrm{t} / \mathrm{ha})$, des racines $(1,96 \mathrm{tC} / \mathrm{ha})$ et des litières $(1,88 \mathrm{tC} / \mathrm{ha})$ est faible, moins de $5 \%$ de carbone totale. Par contre, dans la savane arbustive, le sol constitue le principal réservoir de carbone (74,35 tC/ha). Les autres composantes à savoir les arbustes $(0,66 \mathrm{tC} / \mathrm{ha})$, les herbacées $(3,15 \mathrm{tCha})$, les racines $(1,98 \mathrm{tC} / \mathrm{ha})$ et les litières $(1,34$ tC/ha) ont une contribution très faible, moins de $9 \%$ de la quantité totale de carbone. Les différences observées entre la savane arborée et arbustive sont dues en grande partie à la différence de leur structure et de leur composition floristique. Ces résultats préliminaires contribuent à la compréhension de l'impact des conversions des savanes sur le changement climatique. Ceci permettra une prise de décision dans la gestion durable des savanes et la protection de l'environnement.
\end{abstract}

Mots clés: Phytomasse, Carbone, Réservoir, Savanes soudano-guinéennes, Ngaoundéré, Cameroun

\section{ABSTRACT}

One of the environmental consequences of the modification of savannas is a carbon loss which in turn contributes indirectly to global climate change. In order to evaluate contribution of Adamawa savannas in global climate change, a study on the sequestration of carbon was conducted both in a shrubby and woody savannas of Ngaoundere. The quantity of carbon was estimated in a square plot of $100 \mathrm{~m}^{2}$ for the trees and shrubs, of $1 \mathrm{~m}^{2}$ for grass and $0.0625 \mathrm{~m}^{2}$ for the roots and the soils. The experimental design is a randomized complete block with three replicates. The type of savannas are main treatment whereas the plots are replicates. The results show that the total average of carbon is about $81.48 \mathrm{tC} / \mathrm{ha}$ in shrubby savannas and $118.36 \mathrm{tC} / \mathrm{ha}$ in woody savannas. In the woody savannas, the essential of carbon was stored in the trees $(65.30 \mathrm{tC} / \mathrm{ha})$ and in the soil $(48.37 \mathrm{tC} / \mathrm{ha})$. Whereas in the shrubby savannas, it is based on in the soil. The contribution of shrubs and grass $(0.78 \mathrm{tC} / \mathrm{ha})$, roots $(1.96 \mathrm{tC} / \mathrm{ha})$ and litters $(1.88 \mathrm{tC} / \mathrm{ha})$ is less than $5 \%$ of the global quantity of carbon. Shrubby savannas soil appears to be the main carbon tank $(74.35 \mathrm{tC} / \mathrm{ha})$. The other components of shrubby savannas as shrubs, grass, litters and roots contributed for less than $9 \%$ in the total amount of carbon. The differences noticed between the type of savannas are due to their floristic structure et composition. These preliminary results contribute to the understanding impacts of savannas conversion to global climate change. This would allow to make decision in sustainable management of savannas and environment protection.

Key words: Phytomass, Carbon storage, soudano-guinea savannas, Ngaoundere, Cameroon.

INTRODUCTION

La pression anthropique, à travers les feux de brousse, les mauvaises pratiques agricoles et les coupes anarchiques de bois [1], due à la poussée démographique entraîne une dégradation des savanes [2]. Cette dégradation 
se manifeste par la fragmentation des formations savanicoles, l'érosion de la biodiversité, l'appauvrissement des sols en matière organique et en éléments minéraux, et la diminution de la productivité [3]. Elle conduit également à une libération nette de carbone vers l'atmosphère [4]. Cette situation qui a prévalu depuis des années dans l'indifférence générale, suscite actuellement des nombreuses réactions [5], en particulier celles de la communauté scientifique qui s'accorde sur l'urgence de la mesure à prendre pour coordonner l'exploitation des savanes et mettre au point une politique de gestion durable de ces écosystèmes [6].

En effet, les savanes sont des vastes ensembles hétérogènes qui couvrent environ 20 à $30 \%$ de la surface de la terre [7] et où le rapport des densités des arbres et des herbacées varie suivant le climat, le type de sol et la pression anthropique [8]. L'extension des savanes peut atteindre $40 \%$ des terres [9]. Elles jouent non seulement un grand rôle sur le plan socio - économique des populations riveraines par le ravitaillement en bois de chauffe et d'œuvre, en énergie et fibres, en médicaments, etc., mais également sur celui de l'environnement. En effet, ces vastes ensembles représentent un potentiel réservoir de carbone qui peut contribuer de façon significative au bilan de carbone des sols et de l'atmosphère, et aux flux de celui-ci [10]. Cependant, il existe actuellement une controverse sur le rôle des savanes sur le bilan global du carbone. Sontelles source ou puits du carbone? Les estimations basées sur les changements de l'utilisation des terres indiquent que les savanes sont des sources de carbone [1]. Les utilisations des sols sous les tropiques auraient été responsables d'un cinquième des rejets totaux anthropogéniques de carbone vers les années 1980 [11]. Seurlock et Hall [12] estiment que toutes les savanes peuvent séquestrer du carbone jusqu'à $500 \mathrm{TgC}\left(1 \mathrm{Tg}=10^{12} \mathrm{~g}\right)$, avec 200 à $300 \mathrm{TgC}$ dans les sols, ce qui représente un taux de croissance de 15 à 20\% [13]. Ces derniers résultats sont basés sur l'inventaire de la végétation. Ensemble ces deux approches complémentaires peuvent apporter une meilleure estimation du carbone. Cependant, les savanes ont été longtemps ignorées et la plupart des données disponibles sur le bilan du carbone concernent généralement les écosystèmes forestiers [1]. En effet, les revues de Tiessen et al. [14] soulignent le manque d'évaluation des disponibilités en carbone dans les écosystèmes tropicaux secs en comparaison des zones humides, et la pauvreté des études en Afrique par rapport aux autres régions sèches du monde. Etant donné le contexte économique en Afrique, le contrôle des émissions de gaz à effet de serre n'est sans doute pas une priorité des responsables politiques. Mais la séquestration du carbone par les savanes africaines est une clé pour leur production durable et l'intensification de l'agriculture dans ces écosystèmes.

Les savanes de l'Adamaoua Cameroun, en particulier celles de Ngaoundéré, tout comme les autres savanes du monde ne sont pas épargnées des pressions anthropiques. Car sa population passerait de 490185 habitants en 1987 à 1308866 habitants en 2010 [15]. Cette croissance de la population entraîne un surpâturage [16] et une augmentation des pratiques agricoles extensives ces dernières décennies [15]. A cela s'ajoute la crise économique que traverse le pays avec la dévaluation du monnaie locale en 1994 [17], ce qui a entraîné des prélèvements accrus et anarchiques des bois dans les savanes [18]. En effet, le gaz et le pétrole lampant sont devenus inaccessibles pour la majorité des populations de cette région.

Dans la perspective d'évaluer la contribution des savanes de Ngaoundéré et l'impact de leur dégradation à la fois sur le cycle global de carbone et sur la qualité de la matière organique des sols, il est indispensable d'acquérir des données sur les savanes comme puits ou sources du carbone, car actuellement il y a très peu d'information disponible. Le but de cette étude est d'estimer le carbone stocké dans les compartiments de savanes arbustives et arborées, qui constituent les faciès importants de la végétation naturelle de la région de Ngaoundéré.

\section{MATERIEL ET METHODES \\ Sites d'étude}

Les sites d'étude sont situés dans les savanes de Ngaoundéré, qui elles même appartiennent au plateau de l'Adamaoua, situé entre le $6^{\mathrm{e}}$ et le $8^{\mathrm{e}}$ degré de latitude Nord et le $10^{\mathrm{e}}$ et le $16^{\mathrm{e}}$ degré de longitude Est. Elles s'étendent sur environ $72000 \mathrm{~km}^{2}$, avec une altitude moyenne 
d'environ $1200 \mathrm{~m}$ au centre du Cameroun. Le climat de cette région est du type soudano guinéen d'altitude à tendance humide [19], avec une saison de pluies (avril - octobre) et une saison sèche (novembre - mars). La précipitation moyenne annuelle est de 1500 $\mathrm{mm}$, la température moyenne annuelle est de $23^{\circ} \mathrm{C}$ et l'humidité relative de $70 \%$. Les sols dominants sont les sols faiblement ferrallitiques modaux, développés sur les roches granitiques et les sols ferrallitiques de couleur rouge développés sur les basaltes anciens [20]. La végétation de l'Adamaoua est une savane humide à Daniellia oliveri et Lophira lanceolata $[21,22]$. Cette savane se différencie en savanes herbeuses à Imperata en général d'origine agricole; en savanes arbustives à Annona senegalensis, Bridellia ferruginea, Terminalia glaucescens au feuillage cendré, Hymenocardia acida, Piliostigma thonningii avec la présence notable d'Aframomum latifolium et des tapis d'Andropogonées, plus diversifiés; enfin en savanes arborées à Daniellia oliveri, Combretum molle, Parkia biglobosa, Sygygium guineenese var. macrocarpum, Vitellaria paradoxa. La physionomie de cette végétation est influencée par les actions zoo anthropiques.

Pour caractériser le pool de carbone dans les savanes de Ngaoundéré, deux faciès prédominants de cette végétation ont été sélectionnés, près de l'Université de Ngaoundéré. II s'agit d'une savane arborée et d'une savane arbustive situées sur deux sites de même topographie, de couleur et de texture de sol semblables, mais de physionomie, de structure et de composition spécifique de végétation différentes. Ces sites ne sont pas aussi perturbés. Les sols de ces sites sont du type ferrallitique à texture argileuse. Les autres caractéristiques de sites, leur situation géographique et leur formation végétale dominante sont données dans le tableau 1.

\section{Mesure de la phytomasse}

La détermination de la phytomasse a été réalisée dans trois parcelles de $100 \mathrm{~m}^{2}$ (10 m $\mathrm{x} 10 \mathrm{~m}$ ) dans chaque type de savane. Pour minimiser l'hétérogénéité spatiale, les parcelles étaient peu éloignées les unes des autres. Dans ces parcelles, la biomasse des arbres, arbustes, herbacées, racines et la densité des sols ont été déterminées selon la méthode d'Anderson et Ingram [23]. Un arbre par parcelle de $100 \mathrm{~m}^{2}$ a été trouvé dans la savane arborée. L'utilisation de trois individus pour estimer le carbone contenu dans la biomasse des arbres est faible. Elle pourrait induire une surestimation ou sous estimation de carbone. Pour minimiser ces erreurs, trois parcelles supplémentaires de 2500 $m^{2}(50 m \times 50 m)$ chacune, délimitées dans la savane arborée ont été utilisées pour l'estimation de la biomasse des arbres. Au cours de la détermination de la biomasse, la composition et les noms spécifiques ont été identifiés. Le dispositif expérimental utilisé est un bloc complètement randomisé à trois répétitions. Les types de savanes (sites) sont les traitements et les parcelles ou sousparcelles sont des répétitions.

Tableau 1 : Caractéristiques physiques et pédologiques des sites

\begin{tabular}{lll}
\hline & Savane arbustive & Savane arborée \\
\hline Coordonnées géographiques & $13^{\circ} 22^{\prime} \mathrm{E}$ et $7^{\circ} 22^{\prime} \mathrm{N}$ & $13^{\circ} 35^{\prime} \mathrm{E}$ et $7^{\circ} 27^{\prime} \mathrm{N}$ \\
Altitude $(\mathrm{m})$ & 1112 & 1148 \\
Végétation & Arbustes et herbacées & Arbres, arbustes et herbacées \\
Sol* & Ferrallitique sur roche & Ferrallitique sur roche mère \\
& mère granitique & basaltique \\
Texture* & argileuse & argileuse \\
pH du sol: $0-10 \mathrm{~cm}^{*}$ & 7,28 & 6,78 \\
pH du sol: $10-25 \mathrm{~cm}^{*}$ & 8,5 & 7,8 \\
* Source: Mapongmetsem $2005[30]$. & &
\end{tabular}

Phytomasse aérienne

La biomasse des arbres a été estimée dans les parcelles de $2500 \mathrm{~m}^{2}$, en incluant les trois individus des parcelles $100 \mathrm{~m}^{2}$. L'on a considéré comme arbre tout ligneux dont le diamètre à hauteur de poitrine (DHP) est supérieur ou égal 
à $10 \mathrm{~cm}$ et la hauteur supérieure ou égale à 2 $\mathrm{m}$. Les diamètres du tronc à hauteur de poitrine (à 1,3 $\mathrm{m}$ du sol) et les hauteurs de tous les arbres ont été mesurés respectivement à l'aide d'un mètre ruban et d'un clinomètre.

La biomasse des arbres a été estimée par la méthode indirecte, en utilisant un modèle allométrique, prenant en compte les paramètres de l'arbre (son DHP et sa hauteur). Parmi les équations utilisées, celle d'Anderson et Ingram [23] a été retenue, car elle a été développée dans les conditions climatiques (dont la moyenne annuelle de pluviométrie varie de $1500-4000 \mathrm{~mm}$ ), incluant celle de l'Adamaoua $(1200-2000 \mathrm{~mm})$ et le coefficient de détermination entre la biomasse des arbres et leurs deux paramètres (DHP et hauteur) est hautement significatif $\left(R^{2}=0.987\right)$.

$B(\mathrm{Kg})=\exp \left(-3,1141+0,9719 * \operatorname{Ln}\left(D^{2 *} \mathrm{H}\right)\right)$ Où $B$ est la biomasse des arbres en kilogramme, D est le DHP (cm) et $\mathrm{H}$ est la hauteur de l'arbre (m). Cette biomasse a été ensuite exprimée en tonne par hectare $(\mathrm{t} / \mathrm{ha})$.

La biomasse des arbustes et la masse de litière au sol ont été estimées dans les parcelles de $100 \mathrm{~m}^{2}(10 \mathrm{~m} \times 10 \mathrm{~m})$. Les arbustes ont été inventoriés. Leurs noms, leurs diamètres au niveau de première branche et leurs hauteurs ont été enregistrés. Ils ont ensuite été coupés à ras du sol à l'aide d'une machette et séparés par fraction (feuilles et bois). Les masses humides totales de chaque fraction ont été déterminées à l'aide d'une balance (Sartorius ISO 9001). Un sous - échantillon de chaque fraction a été pris et sa masse humide déterminée par pesé. II servira à estimer la teneur en eau des fractions des arbustes et à faire des analyses complémentaires en laboratoire.

Les litières des arbustes ont été ramassées dans chaque parcelle et triées pour leur débarrasser des débris divers. Leur masse humide a été déterminée comme précédemment et un sous - échantillon a été pris.

La biomasse des herbacées a été estimée dans cinq sous-parcelles de $1 \mathrm{~m}^{2}(1 \mathrm{~m} \times 1 \mathrm{~m})$, délimitées à chaque sommet et au centre de la parcelle de $100 \mathrm{~m}^{2}$. Toutes les espèces qui sont à l'extérieur de la sous-parcelle, mais qui y sont enracinées ont été échantillonnées. Par contre, les espèces enracinées à l'extérieur sont écartées de l'échantillonnage. Toutes les herbacées faisant parties de l'échantillonnage ont été inventoriées, puis coupées à ras du sol. Ces échantillons ont été séparés en composante «vivante» et en composante «morte» (litière). La masse totale de chaque composante a été déterminée par pesé et un sous-échantillon a été pris comme précédemment.

\section{Phytomasse souterraine}

La biomasse racinaire a été estimée dans des placettes carrées de $25 \mathrm{~cm}$ de côté, au centre de chaque sous - parcelle. Des blocs de sol de $62510^{-5} \mathrm{~m}^{3}(0,25 \mathrm{~m} \times 0,25 \mathrm{~m} \times 0,10 \mathrm{~m})$ et de 9375 $10^{-6} \mathrm{~m}^{3}(0,25 \mathrm{~m} \times 0,25 \mathrm{~m} \times 0,15 \mathrm{~m})$ ont été extraits à deux niveaux de profondeur: $0-10 \mathrm{~cm}$ et $10-25$ $\mathrm{cm}$. Ces blocs préalablement pesés, ont été tamisés à l'aide d'un tamis à maille de $1 \mathrm{~mm}$, puis les racines ont été manuellement triées et séparées en deux classes selon leurs diamètres [24]: racines fines $(<2 \mathrm{~mm})$ et petites racines (2 $\leq x<5 \mathrm{~mm}$ ). Ces deux groupes ont été ensuite pesés. Avant le tamisage, un sous-échantillon de sol a été pris et pesé pour calculer sa teneur en eau et sa densité apparente.

Calcul de la masse sèche totale de la végétation et la densité apparente des sols

Tous les sous - échantillons (feuilles, bois, litières, herbes, sol) et les échantillons des racines ont été ramenés au laboratoire dans des sacs en papier pour être séchés à $60^{\circ} \mathrm{C}$ dans une étuve pendant 48 heures, c'est-à-dire jusqu'à atteindre une masse sèche constante.

Les teneurs en eau ( $\mathrm{T}_{\mathrm{e}}$ en \% par rapport à la masse sèche) de tous les sous - échantillons ont été calculées selon la formule suivante:

$$
\mathrm{T}_{\mathrm{e}}=((\mathrm{MH}-\mathrm{MS}) / \mathrm{MS}) \times 100
$$

Où $\mathrm{MH}$ est la masse humide et MS est la masse sèche.

A partir des teneurs en eau des souséchantillons (végétation et sol), les masses sèches totales ont été calculées comme suit:

MST= $100 \times$ MHT / (100 + Te), où MST est la masse sèche totale et MHT est la masse humide totale.

A partir des MST des échantillons du sol, la densité apparente du sol (D) a été calculée comme suit:

$D=M S T /\left(L^{2} \times h\right)$, où L est le côté du bloc du sol $(25 \mathrm{~cm})$ et $\mathrm{h}$ est la hauteur du bloc ou la profondeur du trou (10 ou $15 \mathrm{~cm}$ ). 
Les masses sèches totales appelées phytomasses ont été exprimées en tonne par hectare (t/ha) et la densité apparente du sol en kilogramme par mètre cube $\left(\mathrm{kg} / \mathrm{m}^{3}\right)$.

\section{Analyses chimiques}

Tous les sous - échantillons d'une fraction végétale (feuilles et bois) et d'une composante de la végétation (herbes et litières) ou d'une catégorie des racines et les sous - échantillons des sols d'une parcelle sont groupés en un seul échantillon. Ce qui donne 60 échantillons qui se repartissent comme suit: six échantillons (2 sites $x 3$ répétitions) de feuilles, de litières, d'herbes et de bois, et 24 échantillons de racines (2 sites $x 2$ diamètres $x 2$ profondeurs $x 3$ répétitions) et 12 échantillons de sol $(2$ sites $x 2$ profondeurs $x$ 3 répétitions). Ces échantillons, après réduction en poudre à l'aide d'un broyeur Micro Hammer Mill Culatti, muni d'un filtre de $1 \mathrm{~mm}$, ont été analysés au laboratoire du Département des Sciences Alimentaires et Nutrition (SAN) de I'Ecole Nationale Supérieure Agro-Industrielle (ENSAI) de Ngaoundéré. Les concentrations de carbone ont été déterminées par la méthode colorimétrique [25]. Pour les arbres, la valeur de la concentration en carbone (45\%) couramment admise [26] a été utilisée.

\section{Estimation de la quantité du carbone}

A partir de la masse sèche totale (MST) de la végétation (fraction, composante ou catégorie), de la densité du sol (D) et leurs concentrations en carbone, les quantités en carbone ont été calculées comme suit:

végétation,

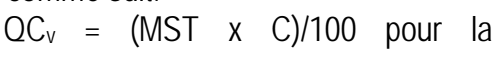

$$
\mathrm{QC}_{\mathrm{s}}=(\mathrm{D} \times \mathrm{C} \times \mathrm{d}) / 100 \text { pour le sol }
$$

Où $\mathrm{QC}_{v}$ est la quantité de carbone de la végétation, exprimée en t/ha, $\mathrm{QC}_{\mathrm{s}}$ est la quantité de carbone du sol, d est la profondeur du sol et $C$ est la concentration en carbone (exprimée en pourcentage de la masse sèche).

\section{Analyses statistiques}

Des analyses des variances suivies de la comparaison des moyennes par le test LSD (Least Significant Difference) à $5 \%$ ont été utilisées pour comparer les paramètres étudiés (arbres, arbustes, herbacées, racines, litières et sols). Des tests t de Student ont été également utilisés pour comparer les deux types de savanes.

\section{RESULTATS}

\section{Composition spécifique et structure des savanes}

La composition spécifique des savanes arborée et arbustive est présentée dans le tableau 2. La savane arborée est plus riche en espèces (19 espèces) que la savane arbustive (10 espèces). Environ 21 espèces appartenant à quatorze familles ont été identifiées. Toutes ces espèces sont présentes dans la savane arborée, excepté deux arbustes, Gardenia aqualla et Vitex madiensis, alors que dans la savane arbustive, les gros et grands arbres comme Daniellia oliveri, Croton macrostachyus, Lophira lanceolata et Vitellaria paradoxa, ainsi qu'un arbuste, Harungana madagascariensis sont absents. Sur les quatorze familles identifiées cinq sont absentes dans la savane arbustive (Clusiaceae, Euphorbiaceae, Ochnaceae, Polygalaceae et Sapotaceae) et deux seulement sont absentes dans la savane arborée (Rubiaceae et Verbenaceae).

Les savanes arbustives et arborées diffèrent entre elles par la densité, la surface basale, le diamètre et la hauteur de leurs végétations (Tableau 3). Ces valeurs sont relativement plus élevées dans la savane arborée (respectivement 3967 ind./ha; 40,65 m²/ha; $15,93 \mathrm{~cm}$ et $3,17 \mathrm{~m}$ ) qu'en savane arbustive (2933 ind./ha; $9,4 \mathrm{~m}^{2} / \mathrm{ha} ; 5,81 \mathrm{~cm}$ et 1,07 m). Car les écarts moyens calculés sont élevés, particulièrement dans les savanes arborées où ils avoisinent la valeur moyenne de chaque paramètre, excepté celui de la surface basale. Ces écarts moyens élevés démontrent qu'il y a une grande différence entre les répétitions (parcelles) pour les valeurs de densité, de diamètre et de hauteur des ligneux et une grande hétérogénéité dans les savanes.

Comme son nom l'indique, la savane arbustive n'est constituée que d'arbustes dont le diamètre et la hauteur varient respectivement de 1,5 à $14,6 \mathrm{~cm}$ et de 0,45 à 1,95 m (Tableau 3). Mais la majorité de ces ligneux ont un diamètre inférieur à $10 \mathrm{~cm}$ et une hauteur inférieure à $1 \mathrm{~m}$ (Figure 1 et 2). Par contre, dans la savane arborée, le diamètre et la hauteur des ligneux varient respectivement de 1,5 à $68,30 \mathrm{~cm}$ et de 0,45 à 15,80 m, avec des densités plus élevées dans les intervalles de 10 à $20 \mathrm{~cm}$ et de 1 à $2 \mathrm{~m}$, respectivement pour le diamètre et la hauteur. 
Tableau 2: Composition spécifique des savanes arbustive et arborée.

\begin{tabular}{llcc}
\hline Familles & Espèces & S. arbustive & S. arborée \\
\hline Anacardiaceae & Lannea schimperi (Hochst. ex A. Rich.) Engl. & $\mathrm{X}$ & $\mathrm{X}$ \\
Annonaceae & Annona senegalensis Pers. & $\mathrm{X}$ & $\mathrm{X}$ \\
Caesalpiniaceae & Daniellia oliveri (Rolfe) Hutch. et Dalz. & - & $\mathrm{X}$ \\
& Piliostigma thonningii (Schumach.) Milne-Rech. & $\mathrm{X}$ & $\mathrm{X}$ \\
Clusiaceae & Harungana madagascariensis Lam. ex Poir. & - & $\mathrm{X}$ \\
& Psorospermum febrifigum Spach & - & $\mathrm{X}$ \\
Combretaceae & Combretum molle R. Br. ex G. Don & - & $\mathrm{X}$ \\
& Terminalia macroptera Guill. et Perr. & $\mathrm{X}$ & $\mathrm{X}$ \\
Euphorbiaceae & Bridellia ferruginea Benth. & - & $\mathrm{X}$ \\
& Croton macrostachyus Hochst. ex Del. & - & $\mathrm{X}$ \\
Fabaceae & Erythrina sigmoidea Hua & $\mathrm{X}$ & $\mathrm{X}$ \\
Hymenocardiaceae & Hymenocardia acida Tul. & $\mathrm{X}$ & $\mathrm{X}$ \\
Mimosaceae & Albizia zygia (DC.) J.F. Macbr. & - & $\mathrm{X}$ \\
& Entada africana Guill. et Perr. & - & $\mathrm{X}$ \\
Myrtaceae & Parkia biglobosa (Jacq.) R. Br. ex G. Don & $\mathrm{X}$ \\
Ochnaceae & Syzygium guineense var. macrocarpum (Engl.) F. White & $\mathrm{X}$ & $\mathrm{X}$ \\
Polygalaceae & Lophira lanceolata Van Tiegh. Ex Keay & - & $\mathrm{X}$ \\
Rubiaceae & Securidaca longepedunculata Fres. & - & $\mathrm{X}$ \\
Sapotaceae & Gardenia aqualla Stapf et Hutch. & $\mathrm{X}$ & - \\
Verbenaceae & Vitellaria paradoxa Gaertn. f. & - & $\mathrm{X}$ \\
\hline
\end{tabular}

$$
\text { X: espèce présente et - espèce absente }
$$

Tableau 3: Structure de la végétation des savanes arborée et arbustive.

\begin{tabular}{lllll}
\hline Paramètres & \multicolumn{2}{c}{ Savane arbustive } & \multicolumn{2}{c}{ Savane arborée } \\
& Moyenne (SE) & Limites & Moyenne (SE) & Limites \\
\hline Densité (ind. ha-1) & $2933(1466,52)$ & $1900-3600$ & $3967(3003,47)$ & $268-8600$ \\
Surface basale $\left(\mathrm{m}^{2} . \text { ha }^{-1}\right)^{\star}$ & $9,40(3,58)$ & $0,02-1,7$ & $40,65(23,83)$ & $0,02-36,62$ \\
Diamètre $(\mathrm{cm})^{\star *}$ & $5,81(2,76)$ & $1,5-14,6$ & $15,93(12,57)$ & $1,5-68,3$ \\
Hauteur $(\mathrm{m})$ & $1,07(0,44)$ & $0,45-1,95$ & $3,17(2,61)$ & $0,45-15,80$
\end{tabular}

SE: écart moyen. *: pour les arbres et les arbustes, ${ }^{\star *}$ : HDP pour les arbres et diamètre au niveau de la première branche pour les arbustes.

Les distributions des classes de diamètre et de hauteur sont différentes entre les deux types de savane (Figures 1 et 2).

Elles ont des formes d'une courbe en cloche avec des pics aux niveaux de $[10-20 \mathrm{~cm}[\mathrm{de}$ diamètre et $[1-2 m[$ de hauteur dans la savane arborée, alors qu'elles ont des formes de $\mathrm{J}$ inversé dans la savane arbustive.

La structure de la végétation diffère entre la savane arborée et la savane arbustive. La végétation de la savane arborée est constituée de trois strates (arborescente, arbustive et herbacée) bien distinctes. La strate arborescente est formée de gros et grands arbres qui atteignent en moyenne $16 \mathrm{~cm}$ de diamètre et dépasse $3 \mathrm{~m}$ de hauteur, donnant l'aspect d'une forêt. La densité des ligneux est très élevée atteignant 4000 individus par hectare. Par contre dans la savane arbustive, les arbres sont quasiment absents ou de petite taille. La végétation n'est constituée que de deux strates (herbacées et arbustives) dont les ligneux n'atteignent guère en moyenne $6 \mathrm{~cm}$ de diamètre et $1 \mathrm{~m}$ de hauteur, avec moins de 3000 arbustes par hectare. 


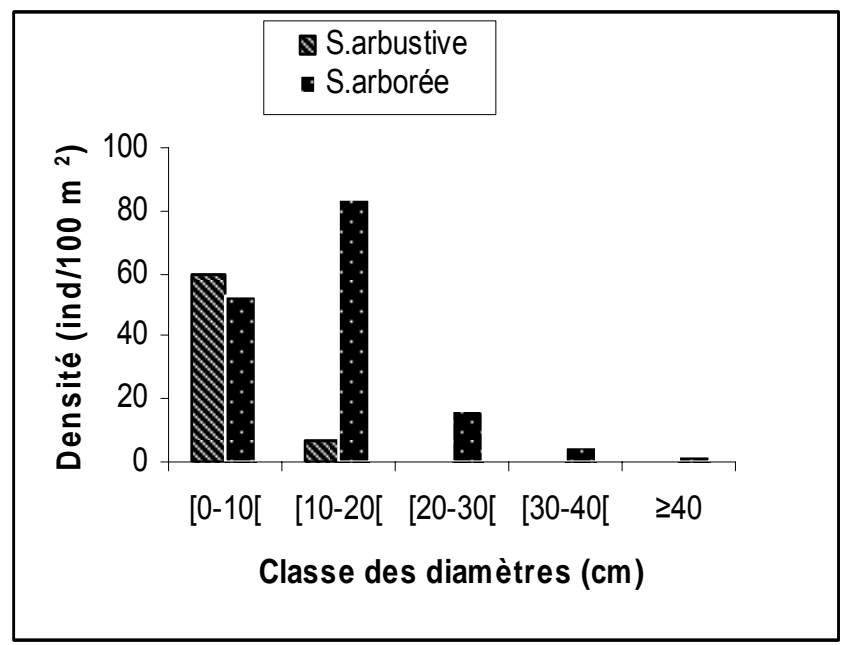

Figure 1: Distribution des classes de diamètre des ligneux dans les deux savanes

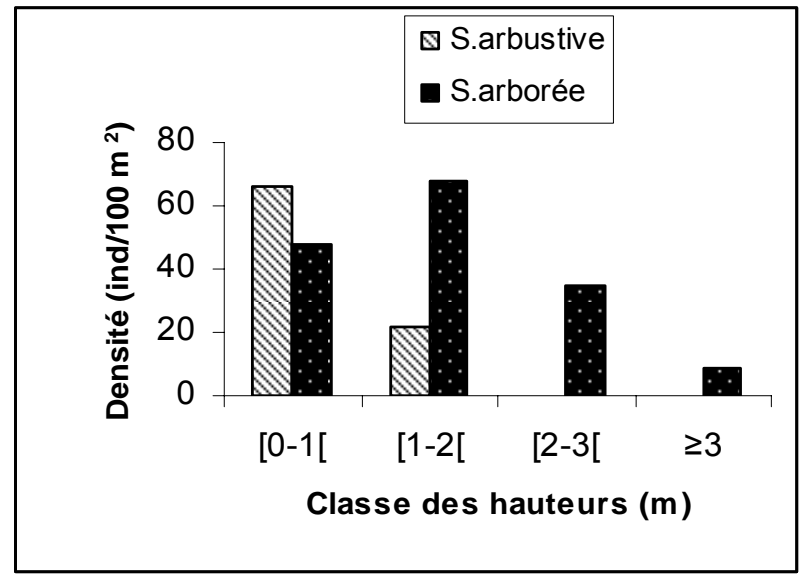

Figure 2: Distribution des classes de hauteur des ligneux dans les deux savanes

\section{Phytomasse}

La phytomasse totale et celles des différentes composantes de la végétation sont présentées dans le tableau 4. Dans la savane arborée, l'essentiel de la phytomasse est constituée par celle des arbres (145,12 t/ha), ce qui représente une contribution de $90,34 \%$ de la phytomasse totale. La contribution des autres composantes de la végétation (arbustes, herbes, racines, litières) est faible, environ 9,66\%. Parmi ces derniers, ce sont les racines $(3,64 \%)$ et les litières $(3,46 \%)$ qui participent le plus à l'édification de la phytomasse et la contribution des herbacées est presque nulle, moins de $0,20 \%$. Par contre dans la savane arbustive, c'est plutôt les herbacées $(7,44$ t/ha) et les racines (5,43 t/ha) qui constituent l'essentiel de la phytomasse (Tableau 4), avec respectivement une contribution de $38,91 \%$ et $28,40 \%$. La contribution des arbustes est faible, environ $12 \%$ et celle de la litière est moyenne $(20,55 \%)$ par rapport aux autres.

\section{Quantité de carbone}

La quantité totale de carbone, celles du sol et de la végétation sont présentées dans le tableau 5. Selon les analyses de variance, la quantité de carbone varie significativement entre les composantes de la végétation et les sols dans les deux savanes (Tableau 5). Mais la comparaison des moyennes par le test de LSD montre que dans la savane arbustive les stocks de carbone ne diffèrent pas significativement à $5 \%$ entre les composantes de la végétation: 
herbacées $(3,15 \mathrm{tC} / \mathrm{ha})$, racine $(1,98 \mathrm{tC} / \mathrm{ha})$, litière $(1,34 \mathrm{tC} / \mathrm{ha})$ et arbuste $(0,66 \mathrm{tC} / \mathrm{ha})$. Dans la savane arborée, la quantité de carbone des arbres (65,30 tC/ha) est significativement supérieure à celles des autres composantes de la végétation, qui ne diffèrent pas significativement entre elles.

La quantité de carbone totale et celle de la litière sont significativement plus élevées dans la savane arborée (respectivement 118,36 et $1,88 \mathrm{tC} / \mathrm{ha})$ que dans la savane arbustive (81,48 et 1,34 tC/ha). Par contre celles des herbacées et du sol sont significativement plus élevée dans la savane arbustive (respectivement 3,15 et $74,35 \mathrm{tC} / \mathrm{ha})$ que la savane arborée $(0,16$ et
48,37 tC/ha). II n'y a pas de différence significative entre les deux savanes en ce qui concerne le carbone des arbustes et des racines.

Dans la savane arbustive, le sol constitue significativement ( $t=9,45$ et $p<0,001$ ) un important réservoir de carbone, avec plus de $91 \%(74,35 \mathrm{tC} / \mathrm{ha})$ de la quantité totale de carbone qui y est stockée (Figure 3). Alors que dans la savane arborée, la majeure partie de la quantité de carbone est stockée à la fois dans les arbres (55,17\% de la quantité totale) et dans le sol $(40,87 \%)$. Ces deux valeurs ne sont pas significativement différentes $(t=0,71$ et $P>0,05)$.

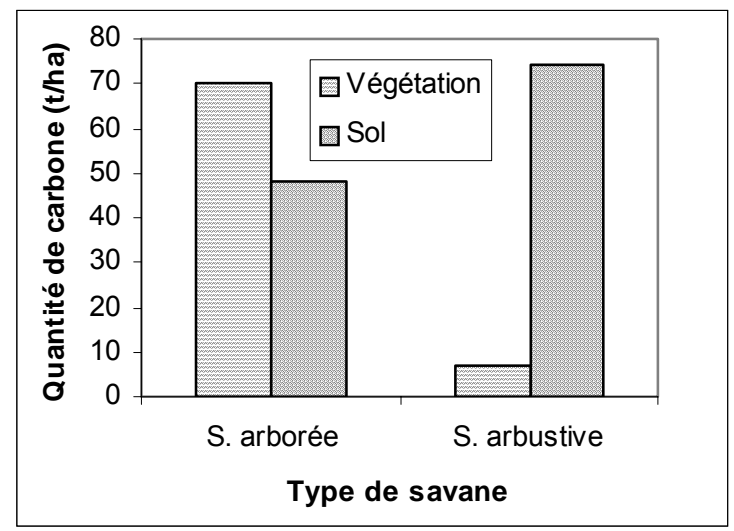

Figure 3: Quantité de carbone stockée dans la végétation et le sol dans les deux savanes.

Tableau 4 : Phytomasse totale et celles des composantes de la végétation (t/ha)

\begin{tabular}{|c|c|c|c|c|c|c|c|c|}
\hline Type de savane & Arbres & & Arbuste & & Herbacées & Racine & Litière & Total \\
\hline & & Feuille & Bois & Total & & & & \\
\hline S. arbustive & 0 & $\begin{array}{l}0,29 \\
(0,20)\end{array}$ & $\begin{array}{l}2,03 \\
(0,65)\end{array}$ & $\begin{array}{l}2,32 \\
(0,06)\end{array}$ & $\begin{array}{l}7,44 \\
(3,03)\end{array}$ & $\begin{array}{l}5,43 \\
(5,16)\end{array}$ & $\begin{array}{l}3,93 \\
(0,36)\end{array}$ & $\begin{array}{l}19,12 \\
(2,21)\end{array}$ \\
\hline S. arborée & $\begin{array}{l}145,12 \\
(106,70)\end{array}$ & $\begin{array}{l}0,95 \\
(0,74)\end{array}$ & $\begin{array}{l}2,87 \\
(1,81)\end{array}$ & $\begin{array}{l}3.83 \\
(0,65)\end{array}$ & $\begin{array}{l}0,30 \\
(0,29)\end{array}$ & $\begin{array}{l}5,84 \\
(0,07)\end{array}$ & $\begin{array}{l}5,56 \\
(0,86)\end{array}$ & $\begin{array}{l}160,64 \\
(101,32)\end{array}$ \\
\hline t Student & & $1,23 \mathrm{~ns}$ & $0,63 \mathrm{~ns}$ & $4,25^{*}$ & $3,31 \mathrm{~ns}$ & $0,14 \mathrm{~ns}$ & $2,93^{*}$ & $4,02^{*}$ \\
\hline
\end{tabular}

Tableau 5: Quantité de carbone (tC/ha) dans la végétation et le sol.

\begin{tabular}{|c|c|c|c|c|c|c|c|c|c|c|}
\hline Type de savane & Arbres & & Arbustes & & Herbacées & Racine & Litière & Sol & $\mathrm{F}$ & Total \\
\hline & & Feuille & Bois & Total & & & & & & \\
\hline S. arbustive & 0 & $\begin{array}{l}0,07 \\
(0,05)\end{array}$ & $\begin{array}{l}0,59 \\
(0,18)\end{array}$ & $\begin{array}{l}0,66 \\
(0,06) a\end{array}$ & $\begin{array}{l}3,15 \\
(1,32) a\end{array}$ & $\begin{array}{l}1,98 \\
(1,96) \mathrm{a}\end{array}$ & $\begin{array}{l}1,34 \\
(0,08) a\end{array}$ & $\begin{array}{l}74,35 \\
(8,42) b\end{array}$ & 94,56 *** & $\begin{array}{l}81,48 \\
(4,20)\end{array}$ \\
\hline S. arborée & $\begin{array}{l}65,30 \\
(48,01) b\end{array}$ & $\begin{array}{l}0,18 \\
(0,17)\end{array}$ & $\begin{array}{l}0,44 \\
(0,38)\end{array}$ & $\begin{array}{l}0,62 \\
(0,10) \mathrm{a}\end{array}$ & $\begin{array}{l}0,16 \\
(0,11) a\end{array}$ & $\begin{array}{l}1,96 \\
(0,26) a\end{array}$ & $\begin{array}{l}1,88 \\
(0,24) a\end{array}$ & $\begin{array}{l}48,37 \\
(10,58) b\end{array}$ & $5,68^{* *}$ & $\begin{array}{l}118,36 \\
(31,20)\end{array}$ \\
\hline t Student & & $2,12 \mathrm{~ns}$ & $0,13 \mathrm{~ns}$ & $0,02 n s$ & $3,33^{*}$ & 0,02 ns & $93,61^{* \star *}$ & $1,47^{*}$ & $4,01^{*}$ & $4,01^{*}$ \\
\hline
\end{tabular}

${ }^{*} p<0,05 ;{ }^{* *} p<0,01 ;{ }^{* *} p<0,001$ et ns non significatif. $F$ résultats des analyses de variances en ligne; les différentes lettres d'une même ligne indiquent que les valeurs sont significativement différentes. 


\section{DISCUSSION}

\section{Estimation de stock de carbone}

La quantité de carbone exprimée en tC/ha est proportionnelle à la phytomasse, car cette quantité a été calculée en multipliant la masse de la végétation ou la densité du sol par la concentration de carbone. II s'en suit que la quantité de carbone et la masse de la végétation ou du sol évoluent dans le même sens.

Les quantités de carbone de la végétation, obtenues dans les savanes soudanoguinéennes de Ngaoundéré (81,48 et 118,36 tC/ha) sont supérieures à celles des savanes soudaniennes sèches (22 - $50 \mathrm{tC} / \mathrm{ha}$ ) et sont dans le même ordre de grandeur que les savanes humides (110 - $126 \mathrm{tC} / \mathrm{ha})$. Elles sont inférieures à celles des forêts tropicales humides du sud Cameroun, qui varient de 199 à 302 tC/ha [26, 27 et 28]. Les savanes soudanoguinéennes de Ngaoundéré sont situées entre les forêts tropicales humides du sud et les savanes sèches du nord Cameroun et constituent l'écosystème de transition. Ces savanes présentent les caractéristiques climatiques semblables à celles des savanes humides.

Dans la savane arborée, la quantité de carbone des arbres est significativement plus élevée que celle des autres composantes de la végétation. Par contre, dans la savane arbustive, c'est plutôt les arbustes et les herbacées qui contiennent plus de carbone. Ces différences entre les deux types de savanes seraient dues non seulement à la différence de leur structure, tel que le type et la densité de la végétation (arbres, arbustes et herbacées), la distribution des classes de diamètre et mais aussi à l'intensité de la pression anthropique qui s'exercent sur les types de savane. En effet, les parcelles situées dans la savane arborée appartiennent à une propriété privée qui est partiellement protégée des pressions zooanthropiques. Le passage de feux de brousse n'est pas régulier; il n'y a pas de surpâturage et les coupes de bois sont pratiquement interdites. Cette protection de la végétation a permis à celle-ci d'atteindre une strate arborescente bien distincte, donnant ainsi une allure de forêt, comme l'ont montré Greg et al. [29]. Par contre, dans la savane arbustive les pressions zoo- anthropiques sont informellement fréquentes et limitent l'évolution de la végétation. Ces pressions constantes maintiennent la physionomie de la savane à une formation basse, constituée de deux strates, formées d'arbustes épars et d'herbacées.

L'importance de la végétation et du sol en tant que puits de carbone est différente entre les deux savanes. En effet, dans la savane arbustive, le sol constitue un important réservoir de carbone, avec plus de $91 \%$ de la quantité totale de carbone qui y est stockée. Alors que dans la savane arborée, la majeure partie de la quantité de carbone est stockée dans les arbres ( $55,17 \%$ de la quantité totale) et dans le sol $(40,87 \%)$. Ceci montre que les mécanismes de la séquestration de carbone dans les deux savanes sont différents et par conséquence, la stratégie de gestion durable dans le maintient du bilan de carbone doivent varier selon le type et le faciès de savane.

\section{Implication dans le bilan global du carbone}

La contribution des savanes humides dans l'évaluation du bilan global du carbone n'est pas négligeable, car les présents résultats comparés à ceux de Ibrahima et al. [27] montrent que le stock de carbone dans ces savanes représente plus de $40 \%$ de celui de la végétation des forêts tropicales camerounaises. Pour une bonne précision, les évaluations du bilan global du carbone doivent tenir compte des savanes, en particulier les savanes humides.

La distribution de carbone dans les compartiments des savanes suggère également que les études de modélisation du cycle global de carbone doivent tenir compte de de l'hétérogénéité des savanes, puisque la stratégie de séquestration de carbone varie selon les faciès de savane.

Pour plus de précision dans l'estimation de carbone dans les savanes soudano-guinéennes de Ngaoundéré, toute évaluation opérationnelle de la disponibilité en carbone et des options de gestion devraient être réalisée non seulement à l'échelle de différents composants de la végétation, de différents compartiments de savane et de différents types d'utilisation de terre, mais également à échelle de l'ensemble des savanes.

Avec la demande accrue en produits vivrières et en énergie, due à la croissance démographique 
et la crise économique, les coupes de bois et les conversions des savanes soudano-guinéennes en cultures prennent une ampleur majeure ces dernières années en Afrique tropicale, en particulier dans la région de Ngaoundéré, Cameroun [18]. La quantité élevée de carbone obtenue dans cette étude suggère que lors des conversions de ces écosystèmes de savane en agriculture, qu'une quantité importante de carbone serait émise dans l'atmosphère. Pour faire face à ces pratiques, des alternatives permettant l'amélioration durable de la productivité des systèmes de production et la limitation des conversions des savanes naturelles en cultures pourraient être recommandées. La fixation de la population dans les savanes et l'intensification durable des cultures sont de moyens de prévention de la déforestation et des pertes ultérieures en carbone dans les savanes naturelles. Pour que ces recommandations soient effectives et rapides, pour la limitation des émissions de carbone gazeux, il faudrait des mesures incitatives conduisant à une source immédiate de revenu pour les paysans.

\section{CONCLUSION}

Les savanes arborée et arbustive diffèrent par la stratégie de séquestration de carbone. Contrairement à la savane arbustive où l'essentiel de carbone est stocké dans le sol, dans la savane arborée, les arbres constituent les principaux réservoirs en carbone. Cette variation du stock de carbone dans les savanes arborées et arbustives est en grande partie due à la différence de leur structure qui est entretenue par l'intensité de la pression zooanthropique. Ces résultats préliminaires contribuent à la compréhension de l'impact des modifications des savanes sur le cycle global de carbone. Ceci permettra une prise de décision dans la gestion durable des savanes et la protection de l'environnement.

\section{BIBLIOGRAPHIE}

1. Houghton R. A., 1995. Effects of land - use change, surface temperature, and $\mathrm{CO}_{2}$ concentration on terrestrial storage of carbon. In Woodwell, G. M., Mackenzie, G. T. (eds.), Biotic Feedbacks in the Global climate system: will the warming speed the warming? Oxford University Press, New York, 333-350.
2. Young M.D. and Solbrig O.T. 1993. Providing an environmentally sustainable, economically profitable and socially equitable future for the world's savannas. In Young M.D. and Solbrig O.T. (eds), The world's savannas: economic driving forces, ecological constraints and policy options for sustainable land use. MAB series, vol. 12, UNESCO, Paris, pp. 321-344.

3. Laurence W., 1998. Dynamique et biomasse des fragments de la forêt amazonienne. Actualités des Forêts tropicales, Bulletin de I'OIBT, 6: 12 - 13.

4. Seiler W. et Crutzen P.J., 1980. Estimated of gross and net fluxes of carbon between the biosphere and the atmosphere from biomass burning. Climatic change 2: $207-247$.

5. Lévêque C., 1994. Le concept de biodiversité: de nouveaux regards sur la nature .NatureSciences 2, 149 - 164.

6.Fujisaka S., Castilla C., Escobar G., Rodrigues V., Veneklaas E.J., Thomas R. et Fisher M., 1998. The effects of forest conversion on annual crops and pastures: estimates of carbon emissions and plant species loss in a Brazilian Amazon Colony. Agric. Ecosyst. Environ. 69: 17-26.

7. Scholes J. et Hall D.O., 1996. The carbon budget of tropical savannas, woodlands and grasslands. In: Breymeyre, A. I., Hall, D., Melilo, J. M., Agren, G. I. (Eds.), Global Change: Effects of Coniferous Forests and Grasslands SCOPE. Wiley, New York, 69100.

8. Skarpe C. 1992. Dynamics of savanna ecosystems. J. Veg. Sci. 3: 293-300.

9. Schimel D.S., Parton WJ, Kittel TG, Ojima DS, Cole CV, 1990. Grassland biochemistry: links to atmospheric processes. Clim. Change 17: $13-25$.

10. Delmas R.A., Loudjani P., Podarre A. et Menaut J.C., 1991. Biomass burning in Africa: an assessment of annually burned biomass. In: Levine J.S.(ed) Global Biomass Burning: Atmospheric, Climatic and Biospheric Implications. MIT Press, Cambridge, MA. 126-132.

11. Schimel D., 1995. Terrestrial ecosystems and the global carbon cycle. Global change Biology, 1: 251-262.

12. Seurlock J.M. and Hall D.O. 1998. The global carbon sink: a grassland perspective. Global Change Biol. 4: 229-233. 
13. Eswaran H., Van den Berg E. Et Reich $P$, 1993. Organic carbon in soils of the world. Soil Sci. Am. J., 57: 192-194.

14. Tiessen H., Feller C., Sampaio EV et Garin P., 1998. Carbon sequestration and turnover in semiarid savannas and dry forest. Climatic change, 40: 105-117.

15. GTZ, 1994. Diagnostic général de la situation de l'environnement dans la province de l'Adamaoua, MINEF, PNUD, 107 p.

16. Rippstein G., 1985. Etude sur la végétation de l'Adamaoua. Evolution, Conservation, Régénération et amélioration d'un écosystème pâturé au Cameroun. Etudes et synthèses de l'I.E.M.V.T. $n^{\circ} 14$, Maisons Alfort, 367p.

17. Eba'a Ayeti R. 2000. TROPFOMS, a decision support model for sustainable management of south-Cameroon's rainforests. Tropenbos-Cameroon Series 2, The Tropenbos-Cameroon Programme, Kribi, Cameroon.

18. Ibrahima A., Mapongmetsem P. M. et Hassan M. 2006. Influences de quelques facteurs zoo - anthropiques sur la phytodiversité ligneuse des savanes soudano - guinéennes de l'Adamaoua, Cameroun. Ann. Fac. Sci. Univ. Yaoundé I, série Sc. de la Nat. et de la vie, 36(3): 65-85.

19. Suchel 1987. Les climats du Cameroun. Thèse de Doctorat d'Etat, Université de Sainte Etienne, T.1, Paris.

20. Humbel F.H., 1971. Carte pédologique de Ngaoundéré 10 à 1/50000.Centre de Yaoundé, Caméroun , O.R.S.T.O.M. Note explicative, $118 \mathrm{p}$.

21. Letouzey R., 1968. Etude phytogéographique du Cameroun. Paris. P Lechevalier (Encyclopédie biologique - LXIX), $511 p$.

22. Yonkeu S., 1993. Végétation des pâturages de l'Adamaoua (Cameroun): Ecologie et potentialités pastorales. Université de Rennes
I. Thèse de doctorat en Sciences Biologiques, France, $207 \mathrm{p}$.

23. Anderson J.M. and Ingram J.S.I. 1993. Tropical soil biology and fertility: $A$ handbook of methods. C. A. B international, UK.

24. Vogt K.A. et Persson H., 1991. Measuring growth and development of roots. Dans: Lassoie J.P. et Hinckley T.M. (eds). Techniques and approaches in forest tree ecophysiology. Boca Raton Etats-Unis.

25. Okalebo J.R., Gathua W.K. et Woomer P.L., 1993. Laboratory methods of soil and plant analyses: a working manual In: Soil Fertility and Biology,Kari, Soil Sciences Society of East Africa, UNESCO-Rosta. 64-65.

26. Kotto-Samé J., Woomer P.L., Moukam A. et Zapfack L., 1997. Carbon dynamics in slashand-burn agriculture and land-use alternatives of the humid forest zone in Cameroon. Agriculture, Ecosystems and Environment, 65: 245-256.

27. Ibrahima, A., Schmidt, P., Ketner, P. and Mohren, G.J.M., 2002. Phytomasse et cycle des nutriments dans la forêt tropicale dense humide du sud Cameroun. TropenbosCameroon Documents 9, $84 \mathrm{p}$.

28. Kanmegne, J., 2004. Slash and burn agriculture in the humid forest zone of southern Cameroon: Soil quality dynamics, improved fallow management and farmers' perceptions. PhD thesis Wageningen University and Research Centre, The Netherlands, $185 \mathrm{p}$.

29. Greg G., Brown S., Massimiliano L. et Singht K.D., 1998. State and change in carbon pools in the forests of tropical Africa. In: Global Change Biology 4: 97 - 114

30. Mapongmetsem P. M. 2005. Phénologie et apports au sol des substances biogènes par la litière de quelques fruitiers sauvages des savanes soudano - guinéennes. Thèse de Doctorat d'Etat ès sciences Biologiques, Université de Yaoundé I, 242p. 